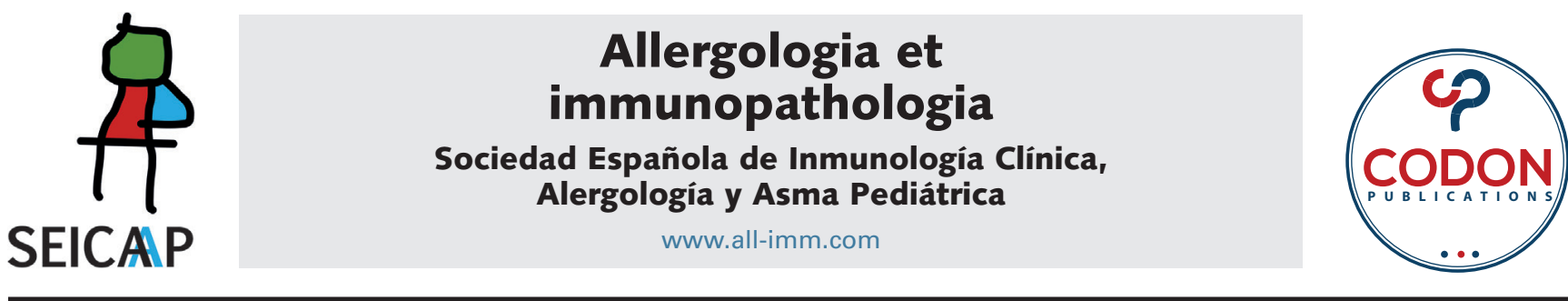

\title{
CME suggestions for pediatricians, allergists, and dermatologists, directed by an online survey on urticaria knowledge
}

\author{
Cesar Fireth Pozo-Beltrán ${ }^{\text {at }}$, Désirée Larenas-Linnemann ${ }^{\mathrm{b}+*}$, \\ Johanna Danielle Cardoza Arteche ${ }^{c}$
}

\author{
${ }^{a}$ Teaching and Research Unit, Hospital de Especialidades Juan María de Salvatierra, La Paz Baja California Sur, México \\ ${ }^{b}$ Research Unit, Médica Sur, Clinical Foundation and Hospital, México City, Mexico \\ cPediatrics Division, Hospital con Especialidades Juan María Salvatierra, La Paz Baja California Sur, Mexico \\ ${ }^{+}$Both authors contributed equally to the development of the paper \\ Presentation at forums: This research was selected for presentation at the Global Urticaria Forum 2018
}

Received 3 August 2020; Accepted 31 October 2020

Available online 2 January 2021

\section{KEYWORDS \\ urticaria; \\ allergists; \\ continuous medical \\ education; \\ dermatologists; \\ pediatricians}

\begin{abstract}
Background: The Mexican Guidelines for the diagnosis and treatment of urticaria have been published. Just before their launch, physicians' knowledge was explored relating to key issues of the guidelines.

Objective: The aim of this study was to investigate the opinion of medical specialists concerning urticaria management.

Methods: A SurveyMonkey ${ }^{\circledR}$ survey was sent out to board-certified physicians of three medical specialties treating urticaria. Replies were analyzed per specialty against the evidence-based recommendations.

Results: Sixty-five allergists (ALLERG), 24 dermatologists (DERM), and 120 pediatricians (PED) sent their replies. As for diagnosis: ALERG 42\% and PED 76\% believe cutaneous mastocytosis, urticarial vasculitis, and hereditary angioedema are forms of urticaria, versus DERM $29 \%$ $(\mathrm{P}<0.005)$. Most of the specialties find that the clinical history and physical examination are enough to diagnose acute urticaria, except DERM 45\% $(\mathrm{P}<0.01)$. DERM 45\% believe laboratory-tests are necessary, as opposed to $<15 \%$ ALLERG-PED $(P<0.005)$. However, PED $69 \%$ did not know that the most frequent cause of acute urticaria in children is infections, versus ALLERGDERM 30\% $(\mathrm{P}<0.005)$. Many erroneously do laboratory testing in physical urticaria and ALLERG 51\%, DERM 59\%, and PED 37\% do extensive laboratory testing in chronic spontaneous urticaria (CSU); many more PED 59\% take Immunoglobulin G (IgG) against foods $(P<0.005)$. More than half of non-allergists do not know about autologous serum testing nor autoimmunity $(P<0.05)$. As for treatment, there were a few major gaps: when CSU was controlled, $>75 \%$ prescribed antihistamines pro re nata, and $>85 \%$ gave first-generation antiH1 for insomnia. Finally, $>40 \%$
\end{abstract}

*Corresponding author: Désirée Larenas-Linnemann. Research Unit, Médica Sur, Clinical Foundation and Hospital, México City, Mexico. Email address: Marlar1@prodigy.net.mx 
of DERM did not know that cyclosporine A, omalizumab, or other immunosuppressants could be used in recalcitrant cases.

Conclusion: Specialty-specific continuous medical education might enhance urticaria management.

(c) 2021 Codon Publications. Published by Codon Publications.

\section{What's New}

Improve your urticaria knowledge: acute urticaria in childhood, first cause is infection. Taking food-specific Immunoglobulin G (IgG) for diagnosis is never recommended. For wheals: mastocytosis and autoinflammatory diseases are differential diagnoses: these are not urticaria and unresponsive to antihistamines. Do not use first-generation antihistamines.

\section{Background}

Over the last years, urticaria specialists have gained new knowledge on several aspects of the pathophysiology of urticaria; consequently, progress in the classification, diagnosis, and treatment of urticaria has been made. The updates of international guidelines have been building on these new concepts. However, guidelines from different regions do not agree on all the details. For example, the European ${ }^{1}$ and World Allergy Organization ${ }^{2}$ experts suggest an allergen-free diet could be helpful in some patients, while the US experts do not make this suggestion, ${ }^{3}$ and vice versa, while US colleagues suggest leukotriene receptor antagonists could be an add-on treatment, European colleagues deleted this option from the preferred medication list. Moreover, locally in Mexico, some specific issues related to urticaria management need to be addressed and adjusted. Thus, the necessity to create national guidelines arose, in which these recommendations of international guidelines were fused and adapted to local reality and needs. The Mexican Guidelines for the diagnosis and treatment of urticaria were created, on which we base the elaboration of this research.

Just before the release of the guidelines manuscript, researchers decided to try to depict how urticaria is generally managed in Mexico and to identify specialty-specific knowledge gaps. The goal of this paper was to establish suggestions on which particular issues, related to the diagnosis and treatment of acute and chronic urticaria, and continuous medical education (CME) could be focused and to develop all this in the context of evidence-based medicine. Moreover, we explored whether there were any specialty-specific topics on which CME should focus to enhance clinical practice among different specialties.

\section{Methods}

We used clinical questions of the 2014 Mexican Guidelines for the diagnosis and treatment of urticaria to obtain opinions of physicians of different specialties (allergists, dermatologists, and pediatricians) concerning the classification, diagnosis, treatment, and physiopathological mechanisms of urticaria. To that end, an online survey with clinical questions was sent out to the members of seven medical societies involved in the creation of the previously mentioned Mexican Guidelines. We present the results of this survey in this paper, against the background of the recommendations and suggestions of the 2014 national guidelines. ${ }^{4}$.

During the transculturation process of the international guidelines for the diagnosis and treatment of urticaria, a group of 35 clinical experts and methodologists created the 2014 Mexican Guidelines for the diagnosis and treatment of urticaria. ${ }^{4}$ This guideline's development group started by determining the goals and objectives of their evidence-based clinical practice guidelines with the SCOPE instrument. Three high-quality internationally available guidelines ${ }^{2,5,6}$ were selected as mother-guidelines with the Appraisal of Guidelines for Research and Evaluation (AGREE-II) instrument. These were used to formulate clue clinical questions, which were answered based on the mother-guidelines' evidence within the cultural and economic context of Mexico. As such, and based on the Grading of Recommendations, Assessment, Development, and Evaluation (GRADE) system, the recommendations and suggestions were developed. A total of 64 questions were grouped into three areas, covering classification and diagnosis of urticaria, treatment, and urticarial physiopathology. Just prior to the launch of the Mexican Guidelines on urticaria, the clinical questions were sent out to the members of the national societies of specialists in dermatology, allergology, and pediatrics through an online survey instrument (SurveyMonkey ${ }^{\circledR}$ ). Most clinical questions were used in their original format; others were somewhat simplified and adjusted for the online survey. The total number of participating societies added up to seven, as some specialties have more than one society.

As the results were collected anonymously, thus making it impossible to trackback to the physician who filled in the questionnaire, in compliance with local legislation neither formal ethics approval nor written informed consent was needed. Even so, the survey started with an invitation to participate and a message stating that by continuing to the next page the physician was consenting for his replies to be analyzed as a group result for final publication.

The clinical questions are presented here with replies per specialty. Statistically significant differences between specialties were calculated with Pearson's Chi-square tests, if necessary, with Yates' correction, using a two-tailed test. We considered $\mathrm{P}<0.05$ to be significant. Only those questions were discussed in which a statistically significant difference between groups was detected. 


\section{Results}

A total of 209 surveys were filled in. Thus, we analyzed the replies of 65 allergists, 24 dermatologists, and 120 pediatricians. The complete file with graphs of all answers per question and specialty could be found in the following: "Replies to Mexican Guidelines on the Diagnosis and Treatment of Urticaria Questions per Specialty” (Supplementary file 1).

\section{Classification and diagnosis of urticaria}

In this section, we describe the replies of the survey related to the suggestions and recommendations for the diagnosis and classification of acute and chronic urticaria. In general, the percentage of correct answers given by three specialties in the completed survey was as follows: allergists $76.7 \%$, dermatologists $65.8 \%$, and pediatricians $69.7 \%$.
Next, questions with a statistically significant difference in correct answers between specialties regarding classification and diagnosis of acute and chronic urticarial in children and adults are presented in Table 1. Less than $30 \%$ of all specialties correctly answered the question referring to the diagnosis of inducible urticaria, where the guidelines recommend against taking laboratory tests or skin prick tests in search of an Immunoglobulin E (IgE)mediated allergy or other pathology. It is important to highlight that cutaneous mastocytosis, urticarial vasculitis, autoinflammatory syndromes, and hereditary angioedema (HAE) should not be considered as forms of chronic urticaria. Nevertheless, unlike the pediatricians, almost half of the allergists and more than one-third of the dermatologists considered these as forms of chronic urticaria; see Figure 1.

Following the diagnosis of chronic spontaneous urticaria (CSU), it is not recommended to take serological tests with food-specific IgG. However, more than $50 \%$ of

Table 1 Frequency of correct answers (\%) among different specialties to clinical questions related to the diagnosis and classification of urticaria.

\begin{tabular}{|c|c|c|c|c|c|c|}
\hline \multirow{2}{*}{\multicolumn{2}{|c|}{ Question }} & \multirow{2}{*}{$\begin{array}{l}\text { Suggested } \\
\text { answer }\end{array}$} & \multicolumn{3}{|c|}{$\begin{array}{l}\text { Percentage of physicians with the } \\
\text { correct answer }\end{array}$} & \multirow{2}{*}{$P$} \\
\hline & & & $\begin{array}{l}\text { Pediatrician } \\
\qquad(n=120)\end{array}$ & $\begin{array}{l}\text { Allergist } \\
(\mathrm{n}=65)\end{array}$ & $\begin{array}{l}\text { Dermatologist } \\
\qquad(n=24)\end{array}$ & \\
\hline $4^{*}$ & $\begin{array}{l}\text { In the classification: Is chemical (aquagenic, cholinergic, } \\
\text { etc.) urticaria separated from physical urticaria? }\end{array}$ & $\mathrm{R}$ : yes & 71 & 46 & 88 & $<0.005$ \\
\hline 5 & $\begin{array}{l}\text { Should cutaneous mastocytosis, urticarial vasculitis, } \\
\text { autoinflammatory syndromes, and hereditary } \\
\text { angioedema be considered subtypes of urticaria? }\end{array}$ & R: no & 24 & 58 & 71 & $<0.005$ \\
\hline 7 & $\begin{array}{l}\text { Are a detailed clinical history and physical examination } \\
\text { enough to diagnose acute urticaria (with or without } \\
\text { angioedema)? }\end{array}$ & R: yes & 85 & 82 & 55 & $<0.01$ \\
\hline 8 & $\begin{array}{l}\text { Should intermittent but recurrent acute urticaria (with or } \\
\text { without angioedema) be considered a form of CU? }\end{array}$ & R: yes & 67 & 87 & 65 & $<0.05$ \\
\hline 9 & $\begin{array}{l}\text { Routine laboratory tests: are they necessary to diagnose } \\
\text { acute spontaneous urticaria? }\end{array}$ & R: no & 89 & 85 & 55 & $<0.005$ \\
\hline 10 & $\begin{array}{l}\text { Is infection the most frequent cause of acute spontaneous } \\
\text { urticaria in children? }\end{array}$ & $\mathrm{R}$ : yes & 31 & 70 & 70 & $<0.005$ \\
\hline 12 & Is acute urticaria more frequent in children than in adults? & R: yes & 89 & 74 & 65 & $<0.01$ \\
\hline 17 & $\begin{array}{l}\text { In physical and cholinergic urticaria, is it useful to } \\
\text { determine the threshold of the triggering stimulus? }\end{array}$ & R: yes & 68 & 84 & 41 & $<0.005$ \\
\hline 18 & $\begin{array}{l}\text { When suspecting contact urticaria, is it useful to do } \\
\text { prick test with the allergen under suspicion to detect } \\
\text { immediate hypersensitivity? }\end{array}$ & S: yes & 72 & 45 & 76 & $<0.005$ \\
\hline 19 & $\begin{array}{l}\text { Should screening tests be taken during the initial } \\
\text { diagnostic approach of CSU? }\end{array}$ & R: yes & 57 & 86 & 88 & $<0.005$ \\
\hline 21 & $\begin{array}{l}\text { Is it advisable to take serological tests of food-specific IgG in } \\
\text { search of the causal diagnosis or triggering factors of CSU? }\end{array}$ & R: no & 40 & 90 & 59 & $<0.005$ \\
\hline 23 & $\begin{array}{l}\text { If the sole manifestation of CSU is angioedema in patients } \\
\text { who are not taking any triggering drugs, is it mandatory to } \\
\text { take laboratory studies to rule out hereditary angioedema? }\end{array}$ & R: yes & 65 & 82 & 82 & $<0.05$ \\
\hline 24 & $\begin{array}{l}\text { Are there any specific details in the medical history or } \\
\text { physical examination that might be a clue to a cause } \\
\text { or a triggering factor for CSU? }\end{array}$ & S: yes & 93 & 98 & 0 & $<0.005$ \\
\hline
\end{tabular}

*Only questions with differences between the specialties in the frequency of correct answers are presented.

CSU: chronic spontaneous urticaria; CU: chronic urticaria. 


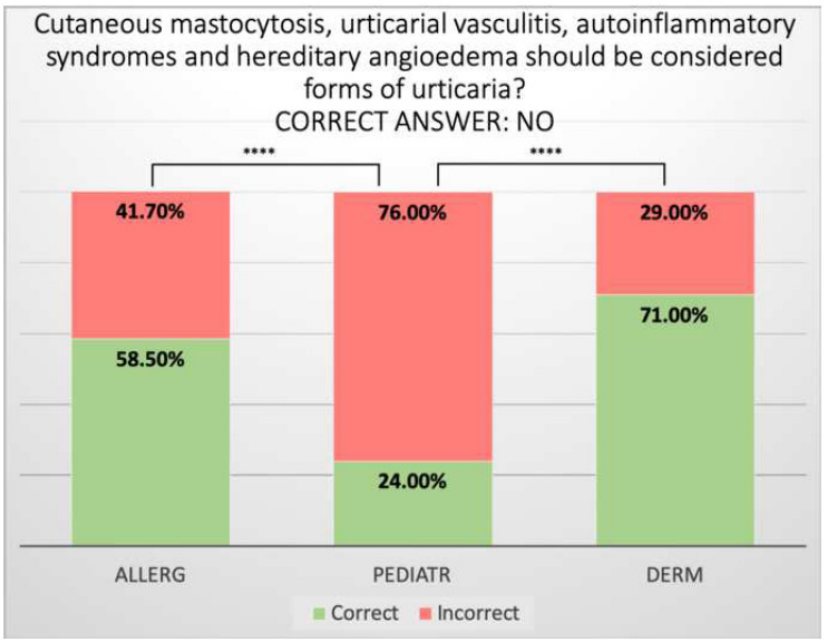

Figure 1 The frequencies of medical specialists' answers regarding cutaneous disorders resembling urticaria. The differences between specialties were analyzed with Pearson's Chi-square test or 2-tailed Fisher's exact probability test (small numbers). Allergists, $n=65$; pediatricians, $n=120$; dermatologists, $n=24$.

\section{Is it advisable to take food-specific lgG in search of the causal diagnosis or triggering factor of chronic spontaneous urticaria? CORRECT ANSWER: NO}

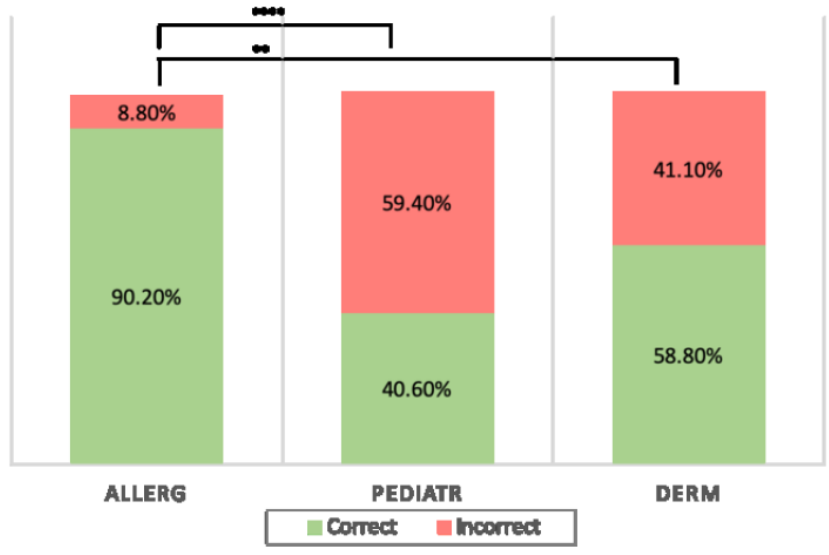

Figure 2 The frequencies of medical specialists' answers regarding the use of serological tests for causal diagnosis or triggering factors of chronic spontaneous urticaria (CSU). The differences between specialties were analyzed with Pearson's Chi-square test or 2-tailed Fisher's exact probability test (small numbers). Allergists, $n=65$; pediatricians, $n=120$; dermatologists, $\mathrm{n}=24$.

the pediatricians and more than $40 \%$ of the dermatologists wrongly considered it as a viable diagnostic test; see Figure 2.

Figure 3 shows in detail the signs and symptoms of the skin lesions that lead different specialties to think that taking a skin biopsy might be recommendable in patients with urticaria. The three specialties agreed, in a higher percentage, to take a skin biopsy when patients present systemic symptoms, but several other alarm signs of the lesions were missed by quite a number of responders.

Subsequently, in the questions without a statistically significant difference between specialties, it is important
In patients with lesions that suggest urticaria, is it advisable to take a skin biopsy if they are.... ?

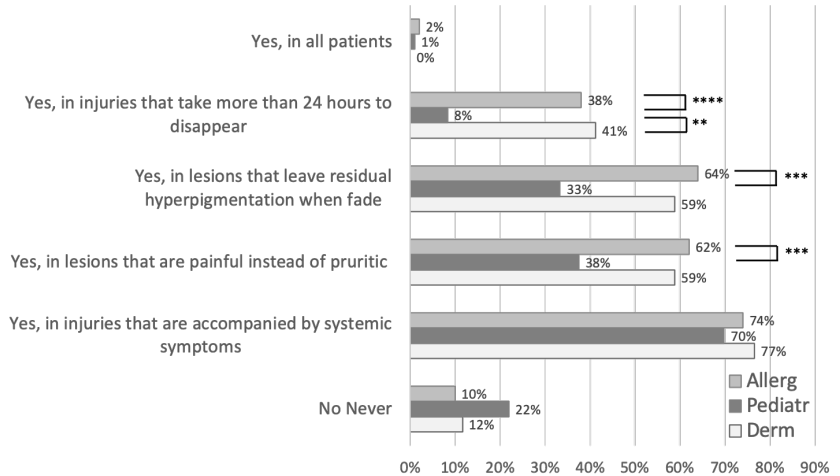

Figure 3 The frequencies of medical specialists' answers regarding the indications for taking a skin biopsy. The differences between specialties were analyzed with Pearson's Chi-square test or 2-tailed Fisher's exact probability test (small numbers). Allergists, $n=65$; pediatricians, $n=120$; dermatologists, $n=24$.

to emphasize that a high proportion of physicians in the three groups did not answer them correctly. Most incorrect answers were given in the Diagnosis and Treatment section.

Regarding the diagnosis of inducible urticaria and CSU, the guidelines recommend against taking laboratory tests or skin prick tests. Nonetheless, the three groups answered this incorrectly and performed procedures despite guideline recommendations. Moreover, the guidelines recommend taking anti-IgE antibodies and anti-receptor IgE by patients in whom a specific cause of CSU cannot be found, but the three groups answered otherwise. This is quite understandable, as these tests are generally not available.

\section{Treatment of Acute and Chronic Urticaria}

In this section, we describe the replies of the survey to questions related to the suggestions and recommendations for the treatment of acute and chronic urticaria. In general, following were the percentages of correct answers to survey questions for this section: allergists $78.8 \%$, dermatologists $72 \%$, and pediatricians $74.6 \%$.

Table 2 presents the questions with a statistically significant difference between specialties regarding the treatment of acute and chronic urticaria in children and adults.

It is important to mention that once the patient with chronic urticaria is managed with the daily administration of a second-generation $\mathrm{H} 1$ antihistamine, the guidelines recommend against changing the administration of the antihistamine to an 'as needed' schedule. Nonetheless, more than $70 \%$ of all the specialists considered this practice to be a viable option. In addition, more than $50 \%$ of our specialists answered that in patients who suffer from insomnia due to nocturnal pruritus, administering a first-generation $\mathrm{H} 1$ antihistamine (sedative) might be advisable, but the guidelines suggest against this.

Over the last years, anti-IgE monoclonal antibodies (omalizumab) have changed the quality of life and the progression of patients with disease that is not controlled 
easily with conventional drugs. ${ }^{7,8}$ The guidelines suggest that anti-lgE monoclonal antibodies are useful in chronic recalcitrant urticaria. Questioned about this issue, more than $70 \%$ of the allergists and pediatricians answered correctly. However, many dermatologists were not yet aware of this new treatment modality; see Table 2.

\section{Discussion}

Using an online-based questionnaire (SurveyMonkey ${ }^{\circledR}$ ), we were able to obtain the ideas from different specialists and pediatricians concerning aspects of urticaria management, starting with diagnosis, through classification to treatment. Knowledge gaps were identified in each of these steps of urticaria management when the replies of the survey were analyzed in the light of the locally accepted urticaria guidelines (Mexican Guidelines on Urticaria, 20144) (Table 3), which, in turn, overlap in the majority of concepts with the recently updated international guidelines on urticaria. ${ }^{1}$

When diagnosing urticaria, physicians should consider differential diagnoses and contemplate several other diseases. Hereditary angioedema can be confused with urticaria manifesting itself solely with angioedema, but the cause is completely different from urticaria: in hereditary angioedema the over-production of vasoactive substance bradykinin instead of histamine is the culprit and treatment with antihistamines is useless. ${ }^{9}$ Then some pathologies can debut with hives as the primary cutaneous manifestation, even though they are not urticaria. Some of these, the autoinflammatory diseases, specifically present themselves from childhood onward; ${ }^{10}$ however, only a quarter of the surveyed pediatricians were aware of this presentation. ${ }^{10}$ In addition, a large number of allergists still missed both of these differential diagnoses. That is why guidelines recommend testing routinely for a complete blood count (CBC), C-reactive protein (CRP), and/or erythrocyte sedimentation rate (ESR) for CSU. Again, only just over half of our pediatricians would do this.

On the other hand, almost half of the surveyed dermatologists erroneously wanted to do routine laboratory tests in patients with acute urticaria, stating that in acute urticaria a detailed clinical history and physical examination are generally not enough. The guidelines indicate otherwise; guideline experts consider cost-saving an important issue, thus recommending against routine laboratory testing in acute urticaria. An eventual exception could be a case of suspected food allergy, when a temporal (maximum eight hours) and repetitive relationship exists between the ingestion of food and the onset of hives. Food-specific IgE testing could be in place, by either skin prick testing or serology, only in these rare cases. The most frequent cause of urticaria in childhood, however, is viral infections. Strikingly, more than two-thirds of the pediatricians were not aware of this fact, and $30 \%$ of the other two specialties also did not know about this cause. Even though skin lesions could be quite spectacular, especially when accompanied by angioedema, acute urticaria secondary to a viral infection is a benign, self-limiting disorder and thus needs neither an aggressive treatment nor any specific laboratory work-up.

Table 2 Frequency of correct answers (\%) among different specialties to clinical questions related to the treatment of urticaria.

\begin{tabular}{|c|c|c|c|c|c|c|}
\hline \multirow{2}{*}{ Question } & & \multirow{2}{*}{$\begin{array}{l}\text { Suggested } \\
\text { answer }\end{array}$} & \multicolumn{3}{|c|}{$\begin{array}{l}\text { Percentage of physicians with the correct } \\
\text { answer }\end{array}$} & \multirow{2}{*}{$P$} \\
\hline & & & $\begin{array}{l}\text { Pediatrician } \\
\qquad(\mathrm{n}=120)\end{array}$ & $\begin{array}{l}\text { Allergist } \\
(\mathrm{n}=65)\end{array}$ & $\begin{array}{l}\text { Dermatologist } \\
\qquad(\mathrm{n}=24)\end{array}$ & \\
\hline $39^{*}$ & $\begin{array}{l}\text { For the treatment of acute } \\
\text { urticaria in pregnant women: } \\
\text { what would be the safest oral } \\
\text { antihistamine }(\mathrm{AH}) \text { ? }\end{array}$ & $\begin{array}{l}\text { S: Loratadine } \\
\text { or cetirizine }\end{array}$ & 90 & 84 & 60 & $<0.05$ \\
\hline 44 & $\begin{array}{l}\text { Once one manages to control } \\
\text { the patient with } \mathrm{CU} \text { with daily } \\
\text { administration of a second- } \\
\text { generation } \mathrm{H} 1 \text { antihistamine } \\
\text { (sgAH) : is it advisable to } \\
\text { indicate the administration of } \\
\mathrm{AH} \text { as needed? }\end{array}$ & R: no & 75 & 80 & 24 & $<0.005$ \\
\hline 45 & $\begin{array}{l}\text { In patients with urticaria who } \\
\text { suffer from insomnia due to } \\
\text { nocturnal pruritus: could a } \\
\text { first-generation sedative oral } \\
\text { AH be prescribed? }\end{array}$ & S: no & 17 & 4 & 0 & $<0.05$ \\
\hline 49 & $\begin{array}{l}\text { In patients with recalcitrant } \\
\text { CU: are anti-IgE monoclonal } \\
\text { antibodies useful? }\end{array}$ & S: yes & 76 & 90 & 57 & $<0.05$ \\
\hline
\end{tabular}

*Only questions with differences between the specialties in the frequency of correct answers are presented.

AH: H1-antihistamine; CU: chronic urticaria; CSU: chronic spontaneous urticaria; Ig: immunoglobulin; sgAH: second-generation H1 antihistamine. 
Table 3 Recommendations and suggestions of the 2014 Mexican Guidelines for the diagnosis and treatment of urticaria to be reinforced per specialty.

\begin{tabular}{|c|c|}
\hline Pediatricians & $\begin{array}{l}\text { R: We recommend against considering cutaneous mastocytosis, urticarial vasculitis, } \\
\text { autoinflammatory syndromes and hereditary angioedema as forms of urticaria. } \\
\text { R: We recommend against using laboratory or skin tests to determine IgE-mediated allergy or other } \\
\text { pathology for the causal diagnosis of chronic inducible urticaria. } \\
\text { S: We suggest against using first-generation oral H1 antihistamine in patients who suffer from } \\
\text { insomnia due to nocturnal pruritus. } \\
\text { R: The most frequent cause of spontaneous acute urticaria in children is infection. } \\
\text { R: We recommend against taking serological tests with food-specific lgG in search of the triggering factors } \\
\text { of CSU. } \\
\text { R: Intermittent but recurrent acute urticaria is considered a form of chronic urticaria. } \\
\text { R: We recommend determining the threshold of the triggering stimulus in physical and cholinergic } \\
\text { urticaria. } \\
\text { R: We recommend taking screening tests during the initial diagnostic approach of CSU. } \\
\text { R: We recommend against taking extensive laboratory studies in search of the causal diagnosis or } \\
\text { triggering factors of chronic spontaneous urticaria. } \\
\text { R: We recommend taking laboratory studies to rule out hereditary angioedema in patients who are not } \\
\text { taking any triggering drugs. } \\
\text { S: We suggest doing autologous serum test for casual diagnosis of chronic urticaria. } \\
\text { S: We suggest taking anti-lgE antibodies and anti-lgE receptors in patients in whom the specific cause of } \\
\text { CSU could not be found; however, it is not available in Mexico. } \\
\text { R: We recommend a questionnaire to measure activity or severity of the disease at the time of the first } \\
\text { consultation. } \\
\text { R: We recommend a questionnaire to measure activity or severity of the disease in subsequent } \\
\text { consultations. } \\
\text { R: We recommend second-generation over first-generation antihistamine for acute urticaria. } \\
\text { S: We suggest to double or quadruple the dose only for some second-generation antihistamines in patients } \\
\text { who do not improve or have moderate to severe symptoms. }\end{array}$ \\
\hline Allergists & $\begin{array}{l}\text { R: We recommend against using laboratory or skin tests to determine IgE-mediated allergy or other } \\
\text { pathology for the causal diagnosis of chronic inducible urticaria. } \\
\text { S: We suggest against using first-generation oral H1 antihistamine in patients who suffer from } \\
\text { insomnia due to nocturnal pruritus. } \\
\text { S: We suggest to skin prick test when suspecting contact urticaria. } \\
\text { R: We recommend against taking extensive laboratory tests in search of the causal diagnosis or triggering } \\
\text { factors of CSU. } \\
\text { Chemical urticaria. } \\
\text { R: We recommend against considering cutaneous mastocytosis, urticarial vasculitis, autoinflammatory } \\
\text { syndromes, and hereditary angioedema as forms of urticaria. } \\
\text { S: We suggest taking anti-IgE antibodies and anti-IgE receptors in patients in whom a specific cause of CSU } \\
\text { could not be found; however, these are not available in Mexico. } \\
\text { R: We recommend a questionnaire to measure activity or severity of the disease at the time of first } \\
\text { consultation. }\end{array}$ \\
\hline Dermatologists & $\begin{array}{l}\text { R: We recommend against using laboratory or skin tests to determine lgE-mediated allergy or other } \\
\text { pathology for the causal diagnosis of chronic inducible urticaria. } \\
\text { R: We recommend a questionnaire to measure activity or severity of the disease at the time of first } \\
\text { consultation. } \\
\text { R: We recommend a questionnaire to measure activity or severity of the disease in subsequent } \\
\text { consultations. } \\
\text { R: We recommend against indicating the administration of antihistamine as needed once the patient } \\
\text { is managed with the daily administration of second-generation H1 antihistamine. } \\
\text { S: We suggest against using the first-generation oral H1 antihistamine in patients who suffer from } \\
\text { insomnia due to nocturnal pruritus. } \\
\text { R: We recommend determining the triggering stimulus in physical and cholinergic urticaria. } \\
\text { R: We recommend against taking extensive laboratory studies in search of causal diagnosis or } \\
\text { triggering factors of chronic spontaneous urticaria. } \\
\text { S: We suggest doing autologous serum test for casual diagnosis of chronic urticaria. } \\
\text { S: We suggest taking anti-IgE antibodies and anti-lgE receptors in patients in whom specific cause of CSU } \\
\text { could not be found; however, it is not available in Mexico. }\end{array}$ \\
\hline
\end{tabular}


Table 3 (Continued)

R: We recommend that doing a physical examination and a detailed clinical history are enough to diagnose acute urticaria.

$\mathrm{R}$ : We recommend that intermittent but recurrent acute urticaria is considered a form of chronic urticaria.

$\mathrm{R}$ : We recommend against doing routine laboratory tests to diagnose acute spontaneous urticaria.

$\mathrm{R}$ : Acute urticaria is more frequent in children than in adults.

$\mathrm{R}$ : We recommend against taking serological tests with food-specific IgG in search of triggering factors of CSU.

R: We recommend making a therapeutic trial by stopping ACE inhibitors or NSAIDs for several months.

S: We suggest taking anti-thyroid antibodies and thyroid function tests in patients in whom a specific cause of CSU could not be found.

S: We suggest loratadine or cetirizine in pregnant women for the treatment of acute urticaria.

S: We suggest using ciclosporin A in chronic recalcitrant urticaria.

S: We suggest using anti-IgE monoclonal antibodies in chronic recalcitrant urticaria.

$\mathrm{S}$ : We suggest using immunosuppressants in chronic recalcitrant urticaria.

Note: Bold=most urgent CME messages, gray=less urgent CME messages.

To avoid the often-needless testing in these children, it is important that physicians are aware of this entity.

When hives appear without any specific physical or chemical trigger, and the disease has lasted for longer than six weeks, CSU is the diagnosis. Nowadays, we know that many of these patients suffer from autoimmunity, type 1 if they have auto-immunoglobulin (Ig) $E$ antibodies, or type 2 if the autoantibodies are of the IgG lineage. Again, an extensive work-up is generally not needed in these patients, as there are no underlying causes to be found for their wheals, other than their autoimmunity. Apart from the routinely recommended $C B C, C R P$, and/or ESR, the guidelines indicate to test further only in case of specific findings in the medical history or in the physical examination, or if CSU is recalcitrant to treatment. Yet, more than half of our survey did indicate extensive testing. Here testing the patients with their own serum (autologous serum testing) might be useful to demonstrate the presence of a histamine-releasing factor in serum, but many surveyed physicians did not distinguish this test.

However, the most important change in practice pattern to be made in the work-up of chronic urticaria is the indication that-apart from the physical trigger-no specific cause should be sought in most of the inducible urticaria variants, as it is the physical factor that causes them. Less than a fifth of our physicians agreed with this fact. An exception to this general rule, however, is cold urticaria that might be caused by infections, comorbidities, or cryoglobulin, among others. Thus, in these patients, one should only look for eventual underlying causes if the patient suffers from cold urticaria or from dual urticaria, in which their lesions not only appear after stimulating with the physical trigger but also spontaneously. Contact urticaria is a less frequent entity in which local contact with an allergen causes the urticaria. Here skin prick testing is in place, but less than half of the allergists expressed this test in their replies.

Finally, the physicians should be able to recognize alarm signs in wheals that indicate the need for taking a skin biopsy to rule out urticarial vasculitis, which can often be a sign of an autoimmune disorder. Here there is considerable room for improvement among non-dermatologists.
Guidelines are clear concerning the main treatment of urticaria: non-sedating second-generation antihistamines, eventually in higher than conventional doses. Generally, those surveyed replied correctly the treatment questions. However, three points could be emphasized here for CME, as less than $80 \%$ of those surveyed were aware of them. First, owing to their lengthy volume of distribution, it takes several days for antihistamines to reach their maximum effect. ${ }^{11}$ Thus, once a patient with CSU is well controlled, continuing low dose as a daily treatment is probably more effective than treatment pro re nata (PRN). The second issue is related to urticaria and insomnia: although first-generation antihistamines cause sedation, generally the guideline experts do not consider their administration to improve patient's night rest as they alter the quality of sleep. First-generation antihistamines have been shown to reduce the rapid eye movement (REM) phase of sleep, ${ }^{12}$ but there is still some disagreement among specialists on this issue. Moreover, very recently, first-generation antihistamines have been linked to dementia because of their anticholinergic action. ${ }^{13}$ Finally, specialists and primary care physicians should be aware that several other treatment options exist for patients with antihistamine-resistant urticaria, such as omalizumab or cyclosporine A, to enhance the timely referral of these patients. Around a third of those surveyed could still learn on this point.

Apart from the global urticaria guidelines of $2018^{1}$ and the local Mexican Guidelines of 2014 on urticaria, ${ }^{4}$ urticaria experts in Spain recently issued a consensus document, developed using the Delphi method, among a panel of 68 experts. ${ }^{14}$ The items and their responses discussed in this Spanish document closely resemble the items questioned in our survey. However, the following two exceptions could be noted: the Spanish colleagues stated that in all patients with suspected CSU, evaluating the thyroid function, and requesting anti-thyroid antibodies should be indicated. Moreover, they affirmed that there is insufficient evidence to recommend the use of anti-leukotrienes in the treatment of CSU. However, our guidelines suggest that anti-leukotrienes could be used as an add-on treatment, especially 
in the patients presenting a flare in their wheals when taking non-steroidal anti-inflammatory drugs (NSAIDs).

\section{Conclusions}

An online survey conducted among physicians of different specialties and primary healthcare colleagues allowed us to detect some knowledge gaps concerning the diagnosis and treatment of urticaria, which might be useful for the correct planning of CME. Moreover, we could distinguish some items with existing specialty-specific inaccuracies, which could be helpful in more specialty-directed CME. After several years of actively promoting and teaching the guidelines, their content has had over 250,000 downloads from the official journal's website and probably still more from other sites where the electronic file is freely available. A repeated survey planned for 2021 might be able to detect a change in the knowledge level of those surveyed, as we have already notice this in informal discussions with colleagues at various congresses.

\section{Competing interests}

None

\section{Funding}

This research did not receive any specific grant from funding agencies in the public, commercial, or not-for-profit sectors. Authors' contributions were pro bono.

\section{Acknowledgments}

We would like to thank those surveyed for their anonymous participation in this study.

\section{Authors' Contribution}

DLL coordinated the Mexican urticaria guidelines and visualized and executed the survey. CFPB gave major support in conducting the survey, and wrote the first MS draft. JDCL supported conducting the survey and the elaboration of results. DLL reviewed the manuscript and molded it into its final version. All authors agreed on the final version of this manuscript and gave consent for its publication in the journal Allergologia et Immunopathologia.

\section{References}

1. Zuberbier $T$, Aberer $W$, Asero R, Abdul Latiff AH, Baker $D$, Ballmer-Weber $\mathrm{B}$, et al. The EAACI/GA (2) LEN/EDF/WAO
Guideline for the definition, classification, diagnosis and management of urticaria. The 2017 revision and update. Allergy. 2018;73:1393-414. https://doi.org/10.1111/all.13397

2. Sanchez-Borges M, Asero R, Ansotegui IJ, Baiardini I, Bernstein JA, Canonica GW, et al. Diagnosis and treatment of urticaria and angioedema: A worldwide perspective. World Allergy Organ J. 2012;5:125-47. https://doi.org/10.1097/ WOX.0b013e3182758d6c

3. Bernstein JA, Lang DM, Khan DA, Craig T, Dreyfus D, Hsieh F, et al. The diagnosis and management of acute and chronic urticaria: 2014 update. J Allergy Clin Immunol. 2014;133:12707. https://doi.org/10.1016/j.jaci.2014.02.036

4. Larenas-Linnemann D, Medina-Avalos MA, Ortega-Martell JA, Beirana-Palencia AM, Rojo-Gutierrez MI, Morales-Sanchez MA, et al. Mexican guidelines on the diagnosis and treatment of urticaria. Rev Alerg Mex. 2014;61(Suppl 2):S118-93.

5. Zuberbier T, Aberer W, Asero R, Bindslev-Jensen C, Brzoza Z, Canonica GW, et al. The EAACI/GA LEN/EDF/WAO guideline for the definition, classification, diagnosis, and management of urticaria: The 2013 revision and update. Allergy. 2014;69:86887. https://doi.org/10.1111/all.12370; https://doi.org/10.1111/ all.12313

6. Powell RJ, Du Toit GL, Siddique N, Leech SC, Dixon TA, Clark AT, et al. BSACl guidelines for the management of chronic urticaria and angio-oedema. Clin Exp Allergy. 2007;37:631-50. https://doi.org/10.1111/j.1365-2222.2007.02678.x

7. Maurer $M$, Kaplan A, Rosen $K$, Holden $M$, lqbal $A$, Trzaskoma BL, et al. The XTEND-CIU study: Long-term use of omalizumab in chronic idiopathic urticaria. J Allergy Clin Immunol. 2018;141:1138-9 e7. https://doi.org/10.1016/j.jaci. 2017.10.018

8. Kaplan A, Ledford D, Ashby M, Canvin J, Zazzali JL, Conner E, et al. Omalizumab in patients with symptomatic chronic idiopathic/spontaneous urticaria despite standard combination therapy. J Allergy Clin Immunol. 2013;132:101-9. https://doi. org/10.1016/j.jaci.2013.05.013

9. Cicardi M, Zuraw BL. Angioedema due to bradykinin dysregulation. J Allergy Clin Immunol Pract. 2018;6:1132-41. https:// doi.org/10.1016/j.jaip.2018.04.022

10. Sag E, Bilginer $Y$, Ozen S. Autoinflammatory diseases with periodic fevers. Curr Rheumatol Rep. 2017;19:41. https://doi. org/10.1007/s11926-017-0670-8

11. Church MK, Maurer M. H(1)-antihistamines and urticaria: How can we predict the best drug for our patient? Clin Exp Allergy. 2012;42:1423-9. https://doi.org/10.1111/ j.1365-2222.2012.03957.x

12. Unno K, Ozaki T, Mohammad S, Tsuno S, Ikeda-Sagara M, Honda $\mathrm{K}$, et al. First and second generation $\mathrm{H}(1)$ histamine receptor antagonists produce different sleep-inducing profiles in rats. Europ J Pharmacol. 2012;683:179-85. https://doi. org/10.1016/j.ejphar.2012.03.017

13. Gray SL, Anderson ML, Dublin S, Hanlon JT, Hubbard R, Walker R, et al. Cumulative use of strong anticholinergics and incident dementia: A prospective cohort study. JAMA Intern Med. 2015;175:401-7. https://doi.org/10.1001/ jamainternmed.2014.7663

14. Gimenez-Arnau A, Ferrer M, Bartra J, Jauregui I, LabradorHorrillo M, Frutos JO, et al. Management of chronic spontaneous urticaria in routine clinical practice: A Delphimethod questionnaire among specialists to test agreement with current European guidelines statements. Allergol Immunopathol. 2017;45:134-44. https://doi.org/10.1016/j. aller.2016.06.007 